\title{
Les conflits d'usage dans les espaces ruraux et périurbains. Le cas des infrastructures franciliennes
}

Land-Use Conflicts in Agricultural and Periurban Areas. The case of Public settings in Ile-de-France

\section{Hai Vu Pham, Thierry Kirat et André Torre}

\section{(2) OpenEdition Journals}

Édition électronique

URL : http://journals.openedition.org/economierurale/3606

DOI : $10.4000 /$ economierurale.3606

ISSN : 2105-2581

\section{Éditeur}

Société Française d'Économie Rurale (SFER)

Édition imprimée

Date de publication : 30 novembre 2012

Pagination : 9-30

ISSN : 0013-0559

Référence électronique

Hai Vu Pham, Thierry Kirat et André Torre, «Les conflits d'usage dans les espaces ruraux et périurbains. Le cas des infrastructures franciliennes », Économie rurale [En ligne], 332 | novembredécembre 2012, mis en ligne le 30 décembre 2014, consulté le 10 décembre 2020. URL : http:// journals.openedition.org/economierurale/3606; DOI : https://doi.org/10.4000/economierurale.3606 


\section{Les conflits d'usage dans les espaces ruraux et périurbains Le cas des infrastructures franciliennes}

Hai Vu PHAM • UMR SAD-APT, INRA \& Agroparistech et IRISSO-Paris Dauphine vxphvu@free.fr

Thierry KIRAT • CNRS, IRISSO-Paris Dauphine

thierry.kirat@dauphine.fr

André TORRE • UMR SAD-APT, INRA \& Agroparistech

torre@agroparistech.fr

C et article s'inscrit dans le cadre des travaux qui se développent autour de l'analyse des conflits d'usage de l'espace dans différents champs des sciences sociales : la géographie sociale (Cadoret, 2006 ; Charlier, 1999 ; Darly, 2009 ; Darly et Torre, 2008 ; Lecourt, 2003 ; Melé et al., 2003), la géographie urbaine (Joerin et al., 2005 ; Trudelle, 2003), la sociologie (Bossuet, 2007 ; Melot, 2009) l'économie régionale (Bouba-Olga et Chauchefoin, 2001 ; Kirat et Torre, 2006 ; Caron et Torre 2006) ou l'économie publique (Jeanneaux, 2006 ; Jeanneaux et Perrier-Cornet, 2009). Cette conflictualité révèle les rivalités et les oppositions, souvent organisées et récurrentes, dans les processus de mutation des espaces et exerce un effet en retour sur les transformations et les vocations des territoires (Torre et al., 2006). Son étude permet d'identifier et de comprendre les résistances à des projets, qu'il s'agisse d'une opposition frontale ou qu'elle porte sur des points particuliers. Cela pose la double question des oppositions et des procédures de décision dans le cadre des aménagements de l'espace relatifs à l'extension des zones urbaines, qui se réalise par de nouvelles constructions ou par l'installation de nouvelles infrastructures à destination de la ville. Cet étalement urbain se fait avant tout sur des terres d'origine agricole, en zones périurbaines ou rurales, qui ont vocation à accueillir les infrastructures au service de la ville.
L'étude empirique porte sur la Région Île-de-France, première agglomération française et deuxième agglomération européenne (Gilly, 2007), qui connaît une pression foncière importante, due à l'accroissement de ses activités et à l'étalement urbain qui la caractérise. Les espaces agricoles ou naturels, qui constituent autant de réserves pour l'urbanisation, se voient progressivement remplacés par des habitations ou par des infrastructures de natures diverses, au service de la ville et indispensables à la vie et à la dynamique de la Région, que ce soient des activités de transport (routes, autoroutes, voies ferrées et de tramways), de production et d'acheminement de l'énergie, de traitement des déchets, ou de constructions en lien avec les activités urbaines.

Avec une population de 11 millions d'habitants (Mathieu, 2005) et environ 23 millions de déplacements quotidiens (Orizet, 2003), la croissance de la Région nécessite la création continue d'aménagements et d'infrastructures et la consommation d'espaces jusque-là dédiés à d'autres fonctions, telles que les activités de production, de loisirs, ou de nature. Les trois quarts de la superficie francilienne sont encore occupés par des espaces ruraux, c'est-à-dire des forêts et des espaces agricoles (Slak et al., 2001). Si l'on se réfère au seul coefficient d'occupation des sols, l'Île-de-France demeure une région à dominante rurale, avec des zones écologiques et environnementales fortement protégées. Toutefois, 
les espaces agricoles franciliens connaissent une tendance forte à la contraction, sous l'effet d'une urbanisation périurbaine majoritairement radioconcentrique, mais qui tend à se diffuser de plus en plus loin de la petite couronne (IAURIF, 2001 ; SEGESA/DREIF/DRIAF, 2005).

Le besoin d'espaces urbanisables dans les communes périurbaines franciliennes à solde migratoire positif est indéniable, tout comme la nécessité d'infrastructures à destination de la métropole parisienne. Toutefois, les marges de manœuvre de la poursuite de l'artificialisation des sols et de la création d'aménagements liés à la mobilité domicile-travail et aux services urbains tendent à se réduire. Les raisons ne tiennent pas seulement à la raréfaction des zones disponibles en périphérie de Paris et le prix croissant des ressources foncières non artificialisées, mais aussi à l'intervention des résidents périurbains, qui ont une préférence pour la préservation de leur cadre de vie et des paysages non ou faiblement urbanisés. Dans un tel contexte, souvent qualifié un peu rapidement de Nimby ${ }^{1}$, il est aisé de comprendre pourquoi les projets de création d'infrastructures en Île-de-France rencontrent fréquemment, comme dans d'autres régions confrontées au problème de l'étalement urbain, de vives oppositions.

La section 1 met en évidence les résultats empiriques obtenus pour les conflits concernant les aménagements et infrastructures en Île-de-France, en détaillant leurs caractéristiques et les acteurs engagés, ainsi que leur ancrage géographique et territorial, avant de présenter un cas emblématique de conflit d'infrastructure. La section 2 propose, à la lumière de ces résultats, une lecture analytique et critique du processus décisionnel qui précède les projets d'aménagement et d'infrastructure. Nous mettons en évidence les caractéris-

1. Not in my Backyard, qui stigmatise l'égoïsme de riverains favorables à la construction d'infrastructures, mais surtout pas à côté de chez eux. tiques du mécanisme complexe qui conduit de la décision publique en matière d'infrastructures à la montée des oppositions, ainsi que les imperfections dans le mécanisme de prise de décision publique, qui contribuent à expliquer la présence et la persistance des conflits.

\section{Caractériser et expliquer les conflits d'infrastructures en Île-de-France}

Cette section est consacrée à une présentation des conflits franciliens en matière d'aménagements et d'infrastructures qui trouvent une expression devant la justice administrative durant la période 1981-2005. Les données utilisées sont issues du contentieux administratif localisé en Île-de-France du $1^{\text {er }}$ janvier 1981 au 31 décembre 2005. Elles consistent en des décisions rendues par le Conseil d'État et, à partir de leur création en 1989, par les Cours administratives d'appel de Paris et de Versailles, sur des conflits localisés dans les six départements de l'Île-de-France, Paris étant exclu du champ de l'étude au regard de la spécificité de la ville-capitale et de la volonté d'axer notre étude sur les zones rurales et périurbaines. Nous utilisons des données issues d'un travail de recueil de textes (les décisions de justice), exploitées sous la forme d'une extraction des données pertinentes via un codage des décisions. La base jurisprudentielle Lamyline Reflex $@$ est la principale source utilisée, chaque décision constituant une observation pour la base de données constituée sur le contentieux. Les décisions de justice permettent d'identifier les arguments avancés par les requérants, c'est-à-dire, en termes juridiques, les «moyens » auxquels la juridiction saisie est tenue de répondre.

Les conflits d'infrastructure mis en évidence sont principalement situés dans les espaces périurbains; ils révèlent les oppositions entre les différents usages du sol, ainsi que les actions menées par des groupes de résidents qui désirent préserver 
leur zone d'habitat. Exprimés sous une forme particulière, qui est le langage du droit, les conflits présentés témoignent des préoccupations locales quant aux localisations futures des équipements publics. Ils révèlent que la détérioration du bien-être des riverains est à l'origine des conflits provoqués par les projets de création d'une infrastructure à proximité. Ces oppositions ne manquent pas de fondements objectifs, même si l'on ne peut exclure les conduites opportunistes. Nous commençons par présenter les objets et les acteurs des conflits d'infrastructures, avant d'envisager leur développement géographique sur la période 1975-2005, puis de détailler un conflit type.

\section{Objets et acteurs des conflits}

Les conflits d'infrastructures nécessitent une attention particulière car ils concernent souvent des projets à fort impact sur la population et sur l'environnement, et se révèlent fortement consommateurs de sols. À partir du recensement exhaustif des décisions des Cours administratives d'appel et du Conseil d'État, l'analyse des principaux conflits d'infrastructures dans la Région permet de donner une idée de la conflictualité liée à ce type d'activités dans les espaces ruraux et périurbains. En effet, derrière la mise en forme juridique des conflits, se révèlent des désaccords sur l'allocation des droits d'usage de l'espace.

L'analyse du contentieux montre que les infrastructures contestées touchent souvent plusieurs communes, généralement périurbaines (Pham et Kirat, 2008). Les équipements publics contestés sont projetés ou construits au bénéfice des habitants de ces communes, mais également de ceux des pôles urbains auxquels elles appartiennent, en particulier Paris. Les conflits d'infrastructures s'inscrivent dans des relations territoriales à différentes échelles : un projet localisé a, dans de nombreux cas, une utilité socio-économique à l'échelle régionale, voire nationale.
Le tableau 1 présente les principaux objets des conflits d'infrastructures sur la période 1981-2005. On voit apparaître au premier rang les infrastructures routières, dont les opposants mettent en avant la pollution de l'air, le bruit, la poussière, l'impact paysager, ainsi que la dévalorisation immobilière. Plus concrètement, il s'agit avant tout des oppositions au bouclage de l'autoroute A86 (deuxième périphérique de Paris) et aux aménagements des routes et autoroutes correspondants.

Tableau 1. Les infrastructures les plus contestées en IDF devant les Cours administratives d'appel et le Conseil d'État (1981-2005)

\begin{tabular}{l|c}
\hline Objets des conflits & $\begin{array}{c}\text { Nombre de } \\
\text { décisions }\end{array}$ \\
\hline Infrastructure routière & 36 \\
Établissement public & 17 \\
Infrastructure de transport & 10 \\
\& production d'énergie & 8 \\
Infrastructure aéroportuaire & 4 \\
Infrastructure ferroviaire & 1 \\
Infrastructure fluviale & 1 \\
Infrastructure de télécommunication & 1 \\
Divers & 78 \\
\hline Total & 78 \\
\hline
\end{tabular}

Au deuxième rang apparaît la création d'établissements publics, tels que des stations d'épuration des eaux usées, des hôtels de ville, des casernes (ou gendarmeries), ou des salles polyvalentes communales. La contestation de ces établissements en soi non polluants est principalement menée par des associations dans le souci de protéger des espaces boisés ou naturels menacés par le projet d'aménagement. Ce qui est jugé discutable n'est pas tant l'infrastructure ellemême que le processus d'urbanisation qu'elle pourrait entrainer.

Le cas des infrastructures de production et de transport de l'énergie (électricité et gaz) est un peu différent. La dangerosité de ces équipements est mise en avant, ainsi que les servitudes d'utilité publique qu'ils impliquent et qui nécessitent un espace dédié. Les opposants à ce type d'infra- 
Graphique 1. Positionnement des conflits d'infrastructures en IDF selon leurs intensités

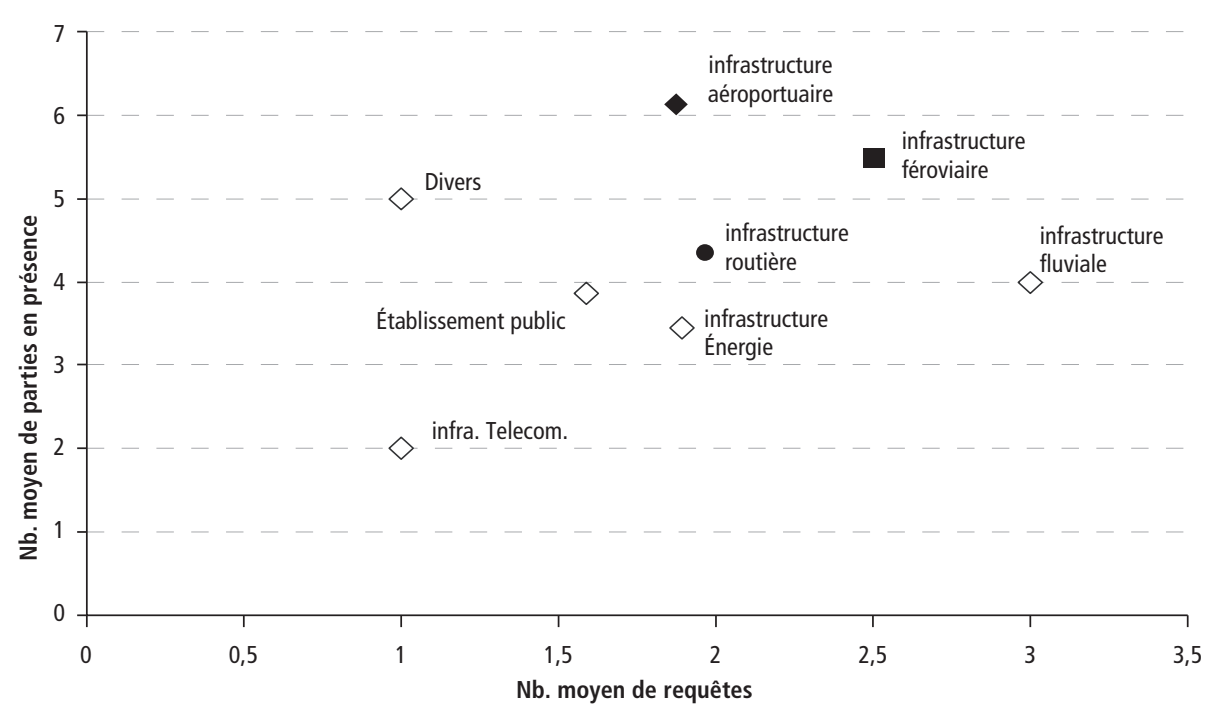

Source : les auteurs

structure sont avant tout des associations de riverains, composées à la fois de propriétaires expropriés et de résidents voisins de l'espace du projet. Les infrastructures aéroportuaires sont le dernier objet emblématique de la Région. En effet, les bruits de décollage et d'atterrissage des avions de nuit font depuis des décennies l'objet de conflits durables entre les riverains, les autorités aéroportuaires et les compagnies aériennes présentes à Roissy et Orly.

On peut émettre l'hypothèse que ce classement, qui accorde une place particulière aux infrastructures de transport de toutes natures, est largement lié au fait que ces dernières parcourent souvent les territoires sur de grandes distances, et donc que leurs conséquences, telles que le bruit associé aux déplacements des avions, affectent des espaces étendus et sont ainsi susceptibles d'incommoder de nombreuses communautés. Il faut également noter que la mesure des conflits par le seul nombre de décisions de justice est partielle, car les opposants peuvent utiliser d'autres moyens que le recours en justice, tels que les manifestations ou pétitions, le recours à la presse ou la violence... Par ailleurs, le juge peut répondre à plusieurs requêtes ou trancher un conflit relatif à plusieurs parties dans le cadre d'un seul jugement.

C'est la raison pour laquelle nous proposons de mesurer l'intensité des conflits par le nombre de parties engagées et le nombre de requêtes traitées dans chaque juridiction (graphique 1). Ces deux variables ont été enregistrées pour chaque arrêt recueilli. Quand le juge décide de répondre à plusieurs requêtes dans un arrêt, il mentionne leurs identifiants dans le texte. Puis il relate l'identité de l'ensemble des acteurs concernés par le jugement : requérants, défendeurs et tiers ${ }^{2}$. Ces informations ont été retenues dans notre base de données. Les conflits les plus intenses - c'est-à-dire ceux qui donnent lieu au plus grand nombre de recours et qui concernent le plus de parties - se retrouvent en haut à droite du graphique 1. Ils sont à nouveau liés à l'aménagement des aéroports, des lignes ferroviaires et routières,

2. Quand un même acteur est présent dans deux requêtes d'un seul conflit, nous ne le comptons qu'une fois. S'il s'engage dans un autre conflit, il sera compté de nouveau comme un nouvel acteur. C'est par exemple le cas des associations de défense de l'environnement présentant plusieurs requêtes. 
RECHERCHES

Hai Vu PHAM, Thierry KIRAT, André TORRE

Tableau 2. Les acteurs et leurs contestations devant la juridiction administrative en matière d'aménagement des infrastructures

\begin{tabular}{|c|c|c|c|c|c|c|c|c|}
\hline $\begin{array}{l}\text { Défendeurs } \rightarrow \\
\downarrow \text { Requérants } \\
\end{array}$ & Entreprises & $\begin{array}{c}\text { Acteur } \\
\text { serv. public } \\
\end{array}$ & $\begin{array}{c}\text { Asso- } \\
\text { ciations }\end{array}$ & $\begin{array}{l}\text { Collectivités } \\
\text { territoriales } \\
\end{array}$ & $\begin{array}{l}\text { Préfet, } \\
\text { ministre }\end{array}$ & $\begin{array}{c}\text { Organisations } \\
\text { professionnelles }\end{array}$ & Particuliers & $\begin{array}{c}\text { Total } \\
\text { Conflits } \\
\end{array}$ \\
\hline Entreprises & & & & 1 & 5 & & & 6 \\
\hline Acteur service public & & & 1 & & & & & 1 \\
\hline Associations & 1 & & 2 & 4 & 67 & & & 74 \\
\hline $\begin{array}{l}\text { Collectivités } \\
\text { territoriales }\end{array}$ & 1 & & 11 & 3 & 33 & 2 & 7 & 57 \\
\hline Préfet, Ministre & & & 13 & 3 & & 2 & 9 & 27 \\
\hline \multicolumn{9}{|l|}{$\begin{array}{l}\text { Organisations } \\
\text { professionnelles }\end{array}$} \\
\hline Particuliers & 2 & & 5 & 4 & 32 & & & 43 \\
\hline
\end{tabular}

et concernent donc des populations importantes. La construction des lignes de TGV par exemple, mobilise plus de 5 parties et fait en moyenne l'objet de plus de 2 requêtes devant le juge administratif. Un recours à l'encontre des projets aéroportuaires engage environ 6 parties et donne lieu en moyenne à 2 requêtes.

Comme le montre le tableau 2, les parties engagées dans les conflits d'infrastructures se répartissent en deux catégories : les acteurs locaux d'une part (associations, collectivités territoriales et particuliers) ${ }^{3}$ et les pouvoirs publics (préfet, ministre $)^{4}$. Si les premiers sont principalement demandeurs de décisions de justice (174 des 208 demandes, soit un taux de $84 \%)$, les seconds sont les plus contestés (137/208 et $66 \%$ des contestations). Ce résultat n'est pas étonnant, dans la mesure

3. Les particuliers n'ont juridiquement intérêt à agir que s'ils subissent des préjudices directs et monétarisables des projets. Cette contrainte juridique limite le recours au juge, qui est coûteux. Des entretiens ciblés montrent que les particuliers sont souvent soutenus par des associations, et que ce statut est parfois un masque emprunté par une association pour contourner des contraintes juridiques concernant son statut.

4. Les collectivités locales relèvent de l'une ou l'autre de ces catégories : en tant qu'acteur local lorsqu'une collectivité conteste une décision publique prise à un autre échelon administratif, en tant que pouvoir public lorsqu'elle prend ou soutient une décision d'aménagement. où les requêtes juridiques liées à l'aménagement des infrastructures consistent généralement à demander soit l'annulation d'un arrêté préfectoral de déclaration d'utilité publique, soit son rétablissement si le tribunal administratif a procédé à son annulation en première instance. La confrontation entre l'intérêt local et l'intérêt général est ainsi ici indéniable. En effet, trois quarts des conflits (152 contestations sur 208) mettent en jeu des confrontations entre acteurs locaux et pouvoirs publics.

\section{La géographie des conflits d'infrastructures Vue globale et longue période}

Les conflits apparaissent dans des espaces et des conditions socio-économiques spécifiques. Il est intéressant de se pencher sur leurs dimensions géographiques, la géographie des conflits d'infrastructures étant un bon indicateur du processus de périurbanisation de l'Île-de-France. La carte 1 montre que les conflits d'infrastructures ne sont pas distribués au hasard dans l'espace francilien : ils se concentrent dans la zone frontalière du grand Paris urbain ; la partie rurale de l'agglomération parisienne semble être faiblement touchée. Les conflits témoignent de la contrainte spatiale à laquelle est confrontée l'agglomération parisienne pour s'agrandir et mettre en place des infrastructures nécessaires à l'aménagement du projet urbain. 
Carte 1. Géographie des conflits d'infrastructure en IDF de 1975 à 2005 (données Lamyline)

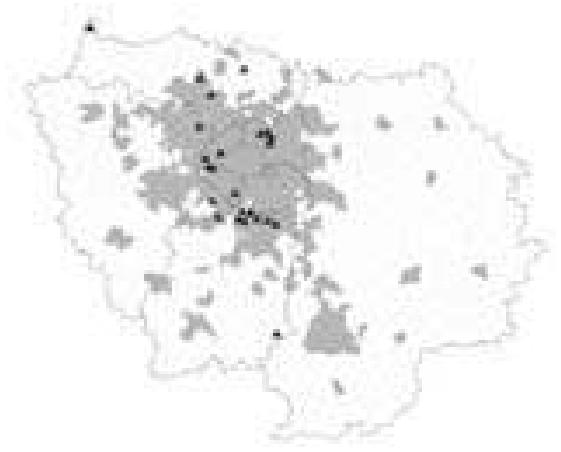

Période 1975 - 1985

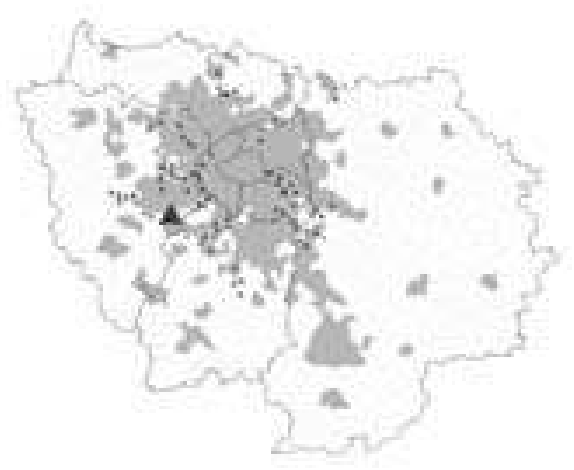

Période 1985 - 1995

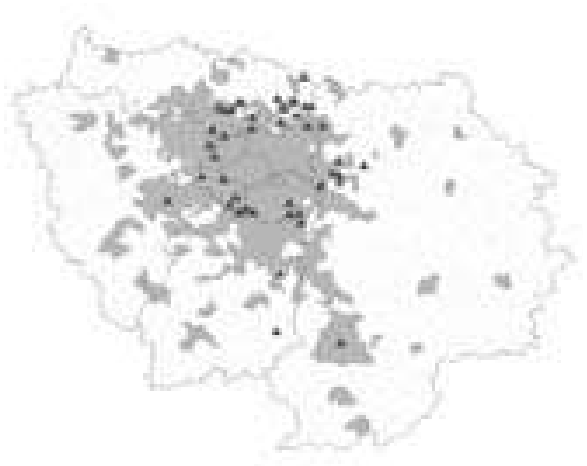

Période 1995 - 2005
La carte 1 suit l'évolution des conflits d'infrastructures sur trois périodes successives $^{5}$ : 1975-1985, 1985-1995, et 19952005. À cette dynamique correspond l'élargissement du fond grisé de la carte, qui désigne les communes possédant une population supérieure à 5000 habitants. Les cartes montrent que, sur ces 30 années, la zone grisée ne s'élargit pas beaucoup, mais que les conflits se multiplient dans des lieux différents, tous situés à la frontière des départements limitrophes de Paris («petite couronne »). Il s'agit de communes périurbaines, à l'interface entre l'agglomération parisienne et les espaces naturels et agricoles, qui représentent encore plus de $50 \%$ de la superficie francilienne. Ces communes ont un rythme d'urbanisation assez élevé (en moyenne plus de 50 autorisations de construction délivrées par an), et sont appelées à devenir urbaines.

Les infrastructures mises en cause sont diverses, mais leur géographie reflète nettement les périodes d'aménagement de l'Îlede-France. Ainsi, la période 1985-1995 est marquée par la construction de l'autoroute A86 et les autoroutes et routes qui lui sont liées, sur des tracés controversés, tandis que la période 1995-2005 révèle l'opposition forte des riverains aux projets d'élargissement des deux aéroports Roissy et Orly. Le bouclage de l'A86 est désormais achevé, mais ses travaux ont commencé en 1968.

Le croisement des résultats issus du contentieux avec des données socio-économiques plus générales révèle que les conflits d'infrastructures se déroulent le plus souvent dans les communes relativement aisées mais où le rythme d'urbanisation est important. Une population communale d'environ 35000 habitants dont $30 \%$ sont des cadres ou exercent une profession intermédiaire ; seulement $15 \%$ d'ouvriers et d'employés ;

5. La période étudiée ici est celle du déclenchement du conflit : nous prenons en compte la date de l'acte à l'origine du conflit et non pas celle du rendu de la décision de la juridiction administrative. 
un nombre effectif de 145 permis de construire délivrés chaque année ; un revenu individuel moyen annuel de $33000 €$; une distance d'une trentaine de $\mathrm{km}$ de Paris : tels sont les traits d'une commune francilienne type - lieu des conflits d'infrastructures. Dans l'ensemble, les problèmes d'urbanisme - délivrance de permis de construire, modification du plan d'occupation des sols ou création d'autres documents d'urbanisme - sont souvent l'objet conflictuel dominant dans les communes plutôt aisées (plus de $30 \%$ de cadres et professions intermédiaires), tandis que l'opposition aux infrastructures se situe fréquemment dans les communes à forte urbanisation ${ }^{6}$. Les conflits liés à la localisation des installations classées, quant à eux, se trouvent dans les communes peu aisées (plus de $30 \%$ d'ouvriers).

Ces résultats rejoignent ceux de Darly (op. cit.) et de Darly et Torre (op. cit.), qui ont analysé les conflits dans les zones rurales et périurbaines de l'Île-de-France à partir de l'exploitation des articles publiés dans la Presse quotidienne régionale (PQR), en l'occurrence le journal Le Parisien. La principale concentration de conflits issus de l'analyse de la PQR se trouve en effet dans l'Ouest Parisien, qui concentre les populations les plus aisées et des communes fortement urbanisées. La mise en parallèle des deux sources (PQR et contentieux administratif) révèle les stratégies différentes des acteurs en matière de déclenchement des relations conflictuelles. Dans les territoires sous pression directe de l'agglomération parisienne, à la frontière urbaine, les stratégies d'affrontement (guerre juridique) se révèlent domi-

\footnotetext{
6. Mesurée par le nombre moyen d'autorisations de constructions délivré chaque année dans une commune. Les communes délivrant en moyenne plus de 50 autorisations par année sont ici considérées comme à fort rythme d'urbanisation, et celles de moins de 50 autorisations comme « faiblement » urbanisées.
}

nantes, alors que dans les zones un peu plus lointaines, c'est plutôt la publicisation par la manifestation publique ou la couverture médiatique qui fait office de sonnette d'alarme à la détection des projets d'aménagement. Il semble y avoir une gradation dans l'engagement dans le conflit, qui part de la mobilisation des médias à l'annonce des projets et se poursuit par le recours à la justice à partir de la mise en place de la procédure de déclaration d'utilité publique. Les projets les plus controversés se localisent toutefois sur le périurbain assez peuplé, à la frontière urbaine et en cours d'urbanisation.

\section{Un conflit type : la déviation de la route $\mathrm{D} 837$ à Maisse (Essonne)}

Examinons, pour illustrer les ressorts internes de cette conflictualité, un cas de conflit caractéristique des décisions d'aménagement et des oppositions qu'elles suscitent en Région Île-de-France. Il s'agit du conflit au sujet de la déviation de la route D837 à Maisse (Essonne), opposant une association de riverains au Préfet sur son caractère d'utilité publique.

La route départementale 837 assure pour le Sud du département de l'Essonne l'important rôle de liaison entre Fontainebleau et l'autoroute A6 à l'Est et la RN 20 à l'Ouest. Elle relie également Ablis avec les autoroutes A10 et A11 via la RN 191. Traversant le cœur de la commune de Maisse dans des conditions jugées incompatibles avec l'important écoulement du trafic routier - étroitesse de la voie, stationnement latéral autorisé et faible largeur des trottoirs dans le centre - elle devient dangereuse pour les piétons et génère de fortes nuisances de bruit et de pollution pour les riverains. Devant cet état de fait, le département de l'Essonne a projeté de réaliser une déviation de la D837 contournant l'agglomération de Maisse.

Cette commune d'environ 3000 habitants, d'une superficie de $22 \mathrm{~km}^{2}$, s'étend au bord de la vallée de l'Essonne. Comme 
le projet ne consiste pas seulement à désengorger son centre-ville, mais également à assurer un passage entre l'autoroute A6 et la N20, il présente un intérêt incontestable pour le département de l'Essonne. Le projet de contournement a été approuvé par le Conseil général en 1999, et déclaré d'utilité publique par le Préfet en avril 2004. Il correspond pourtant à un conflit long d'une vingtaine d'années. En effet, si le désengorgement du centreville s'avère une nécessité pour les habitants de Maisse et leurs voisins, la question de son tracé se révèle rapidement sensible et complexe.

En 1990, le Préfet lance un projet pour détourner la RD837 vers le Sud. Ce tracé suit la frontière de Maisse avec la commune voisine de Gironville-sur-Essonne et traverse une zone relativement peu peuplée. Validé par une déclaration d'utilité publique le 16 mai 1990, avant d'être prorogé par un arrêté préfectoral du 12 mai 1995 à la demande du Conseil général, ce projet est officiellement abandonné le 23 septembre 1999 par une délibération du département, pour des raisons socio-économiques, financières et environnementales. La même année, Maisse entre dans le périmètre du Parc naturel régional du Gâtinais français ${ }^{7}$ et la protection des espaces naturels conduit à l'abandon du tracé Sud. Un nouveau projet est envisagé, cette fois au Nord, où se sont développés entre-temps de nouveaux lotissements résidentiels, occupés par des riverains prévoyant que la déviation passerait au Sud.

Le conflit s'étend alors autour du choix du tracé Nord. D'un côté, les protagonistes de la déviation, à savoir la commune de Maisse, le département de l'Essonne et le Préfet, voudraient réaliser le plus tôt possible la déviation, au nom de l'intérêt général. Le maire de Maisse a même interdit le passage des poids lourds au centre-ville pour assurer la sécurité de ses habitants. De l'autre, les riverains s'opposent vivement à la construction d'une route passant devant leurs propriétés nouvellement construites. L'Association contre la déviation du Nord de Maisse (ADDNM) est créée dans ce contexte : née en 2003 au cours de l'enquête publique, elle regroupe des riverains s'opposant au tracé vers le Nord dans 4 communes: Courdimanche sur Essonne, Boutigny sur Essonne, Maisse et Mily la Forêt. L'objet de cette association (principal opposant tout au long de ce conflit) est l'annulation du projet de déviation vers le Nord.

Pour comprendre l'histoire du conflit, notamment les raisons du choix du tracé Nord, il est nécessaire d'examiner les variantes présentées dans le dossier d'étude d'impact. La carte 2 ci-après présente les 4 variantes $\mathrm{A}, \mathrm{B}, \mathrm{C}$, et $\mathrm{D}$ proposées, qui se distinguent principalement par leurs distances au centre-ville de Maisse. La variante D - la plus courte - passe au Sud de Courty et de la zone de forte densité de population de la commune. Deux variantes médianes - $\mathrm{B}$ et $\mathrm{C}$ - traversent Maisse par la zone résidentielle dans le Nord. Quant à la variante A - la plus longue - elle contourne Maisse par sa frontière avec les communes de Courdimanche-sur-Essonne et de Boutigny-sur-Essonne, et traverse la zone nouvellement urbanisée mentionnée ci-dessus. Longue de $6,5 \mathrm{~km}$, c'est la plus éloignée des habitations.

Finalement, c'est le tracé A, le plus lointain, qui est retenu par l'étude, au motif qu'il a le plus faible impact acoustique et visuel sur les riverains. Il est également évident qu'il s'agit de la solution la moins coûteuse en matière d'expropriation.
7. Le PNR du Gâtinais français couvre 57 communes, dont Maisse et ses communes voisines. 


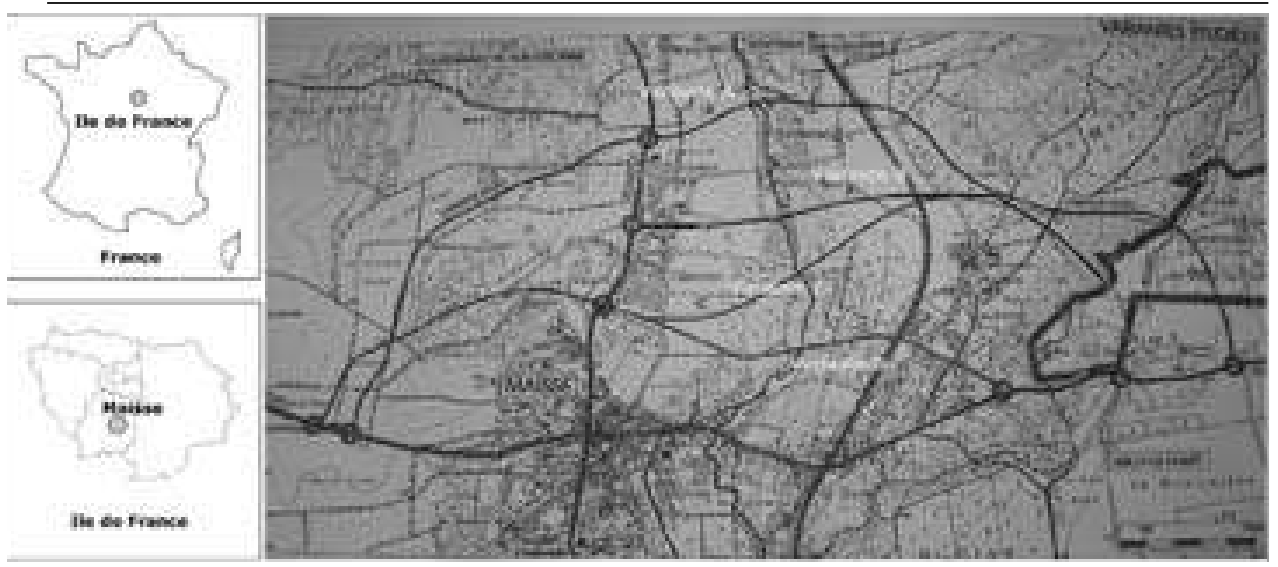

Observons le conflit par une analyse du contentieux $^{8}$. La bataille juridique que mène l'ADDNM à l'encontre du projet a commencée en 2004. Sur ce plan, l'ADDNM a été rejointe par la commune de Courdimanche sur Essonne - également opposée à la déviation sur son territoire. Deux requêtes, respectivement de l'ADDNM et de la commune de Courdimanche, ont été déposées devant le Tribunal administratif de Versailles, qui a statué en leur faveur. Mais le combat ne s'est pas arrêté là, puisque le Conseil général de l'Essonne, le Préfet de l'Essonne, le ministre de l'Intérieur et de l'Aménagement du territoire ont fait appel devant la Cour administrative d'appel de Versailles qui a statué en leur faveur ; l'ADDNM, et la commune de Courdimanche se sont pourvus en cassation devant le Conseil d'État qui a pris une décision de rejet, ce qui a pour effet de confirmer la légalité et l'utilité publique du projet'.

Afin de comprendre les causes du conflit, ainsi que les positions et les revendications des parties en présence, il est important d'entrer dans les arguments présentés. Nous ne retenons pas les motifs de «légalité

8. Dans le cadre de l'étude, nous avons également réalisé des entretiens et un recensement de la presse. Ces sources complémentaires confirment les résultats de l'analyse du contentieux ici présentée.

9. Conseil d'État, Association de défense contre la déviation au Nord de Maisse, 26 janvier 2011. externe » (incompétence de l'acteur du litige, vices procéduraux), pour nous concentrer sur les éléments qui portent sur le fond de l'affaire et éclairent le lien entre conflit et décision publique. Les principaux éléments de contestation sont de trois ordres :

- l'utilité publique, contestée par les opposants au projet ;

- le calcul économique public, les opposants soutenant que ce calcul est erroné ; - l'optimalité de la décision publique. Cette question n'est pas été évoquée en tant que telle mais fait partie des arguments de l'ADDNM, qui soutient que la décision en question n'est pas la meilleure au regard des solutions alternatives.

Sur le premier point, l'argument central des riverains est que différents éléments de nature à modifier le caractère d'utilité publique du projet, en particulier sur le plan environnemental et paysager, n'ont pas été pris en compte dans l'arrêté préfectoral l'approuvant. Cet argument a été favorablement accueilli par le Tribunal administratif, «l'impact paysager et écologique du projet apparaît important sur le site classé de la moyenne vallée de l'Essonne, protégé en raison de sa valeur paysagère et patrimoniale; deux zones naturelles d'intérêt écologique, faunistique et floristique (ZNIEFF) en seraient affectées; une chênaie pinède oligotrophe et un bois de chêne pubescent, qualifiés l'un et l'autre d'intérêt écologique 
exceptionnel par l'étude d'impact, seraient partiellement détruits ; en outre, selon l'étude d'impact, le projet, surplombant les habitations de la vallée, impliqueraient des désagréments visuels pour les riverains. Au total, les atteintes à l'environnement et au paysage impliqués par l'opération paraissent excessifs au regard de l'intérêt de la déviation » (jugement 0404663, 2006).

Mais la Cour administrative d'appel n'a pas partagé ce point de vue. Elle a considéré que « la topographie des lieux marquée par une longue descente dans une rue étroite et animée de village avec un croisement régulé par un feu rouge en milieu de descente et en cour d'agglomération induit un risque élevé d'accidents graves [...] que l'intérêt général attaché à l'objectif de réduction des nuisances pour l'ensemble des habitants de la commune [...] n'est pas contesté ». Cette discussion nous conduit au cœur du problème de l'appréciation qualitative de l'utilité publique, qui peut être formulée en termes d'arbitrage entre un enjeu environnemental et un enjeu de sécurité routière.

Sur le deuxième point - la validité du calcul économique public - l'ADDNM soutient que les calculs économiques ont été basés sur des considérations contestables. En particulier le coût de réalisation du projet serait sous-évalué de $40 \%$ du fait de l'omission de l'inflation dans le taux d'actualisation et de l'oubli d'un carrefour giratoire dans les ouvrages de construction. Le débat porte cette fois directement sur des éléments quantitatifs, puisqu'il s'agit de la sous-estimation du coût du projet. Pour le Tribunal administratif, ces points sont susceptibles de modifier le caractère d'utilité publique du projet car, selon la jurisprudence administrative : « une opération ne peut être déclarée d'utilité publique que si les atteintes à la propriété privée, le coût financier et éventuellement les inconvénients d'ordre social et les atteintes à d'autres intérêts publics qu'elle comporte ne sont pas excessifs eu égard à l'intérêt qu'elle présente ». Le Tribunal administratif a ainsi appliqué la théorie jurisprudentielle dite « du bilan » pour montrer que le bilan du projet sera limité si l'on tient compte des « erreurs » de calcul économique (voir paragraphe II.2., ci-dessous).

La Cour administrative d'appel retient cependant une autre manière de juger ce bilan. Au lieu de discuter le calcul tel qu'il a été invoqué, elle soutient que «ni le coût élevé, ni les inconvénients pour l'environnement et l'agriculture que présente l'opération [...] ne sont d'une importance telle qu'ils aient pour effet de lui (le projet) retirer le caractère d'utilité publique ». Le juge a alors considéré que l'intérêt du projet ne sera pas défini sur un bilan positif du calcul des coûts - avantages, mais encore une fois sur des éléments qualitatifs, qu'il s'agisse de la sécurité routière ou du besoin d'aménagement exprimé par les pouvoirs publics. Il a fait prévaloir alors la sécurité et le trafic routier au-delà du calcul des coûts monétaires. Sa réponse ramène finalement la discussion au deuxième point : l'appréciation qualitative de l'utilité publique. $\mathrm{La}$ théorie du bilan n'a donc pas permis de trancher ce débat. La balance entre des éléments qualitatifs et quantitatifs selon les deux juridictions administratives met en évidence toute la difficulté de juger de la valeur d'un projet en termes d'utilité publique.

Concernant le troisième point, il faut noter que l'ADDNM ne s'oppose pas à la déviation, mais soutient que celle-ci devrait passer au Sud et non pas au Nord. Plus long en distance, le tracé Nord s'annonce non seulement plus coûteux, mais aura également un impact plus important sur le cadre de vie résidentiel que l'itinéraire Sud. L'association évoque ainsi une comparaison des tracés Sud et Nord sur les mêmes critères de choix. Or, cette demande est rejetée par la Cour administrative d'appel, car « au regard au délai écoulé entre l'abandon du projet de contournement par le sud [...] le premier projet (par le sud) ne pouvait être regardé comme un "parti envisagé" au sens des 
dispositions précitées de l'article R.11-3 [nda : du Code de l'expropriation pour cause d'utilité publique] ». Sans doute les tribunaux ne sont pas des lieux de justification d'une décision publique au regard des critères politico-économiques, et la vocation du juge se limite à « répondre juridiquement à des demandes exposants des litiges [...] ou à des requêtes résultant de la lésion d'intérêts » (Kirat et Melot, 2006).

À ce stade, nous constatons que le processus décisionnel ne laisse pas véritablement de place pour mettre en débat l'optimalité d'une décision : le calcul économique mené en amont du projet statue sur son intérêt public, et la procédure d'enquête publique a pour vocation d'en donner confirmation. Il en découle une double limite : d'abord, même si la procédure de consultation du public est organisée formellement, elle ne garantit pas que tous les intérêts s'expriment ; ensuite, le processus ne permet pas l'élaboration ou la discussion d'alternatives.

Les éléments qui précèdent éclairent les origines et les motifs d'une opposition menée pendant de nombreuses années contre les Pouvoirs publics. L'analyse du contentieux permet de douter de la possibilité de vérifier avec impartialité l'optimalité de la décision publique. En particulier, l'enquête publique ne permettant pas d'atteindre une acceptabilité sociale non entachée d'un sentiment d'injustice (Raux et al., 2007), elle provoque oppositions et conflits relatifs aux décisions d'implantations d'infrastructures. Nous argumentons maintenant cette position.

\section{Les projets d'aménagement, la décision publique et les conditions de sa contestation}

Nous avons présenté l'importance des conflits d'infrastructures en Île-de-France, ainsi que leur emprise spatiale et les acteurs qui les animent. L'exemple de Maisse nous a permis de pointer les questions posées par la décision publique, et les oppositions suscitées parfois au niveau local, en raison des incompréhensions qu'elle génère. Sur la base de ces études du contentieux et de notre expérience d'autres conflits d'infrastructures qui se sont déroulés dans différent territoires de l'hexagone, nous tentons maintenant de mettre en évidence les caractéristiques du mécanisme complexe qui conduit de la décision publique à la montée des oppositions, ainsi qu'à l'éventuelle apparition de conflits face à des populations pas toujours disposées à une acceptation sans réserve de projets d'aménagements.

Les acronymes ne manquent pas pour qualifier les oppositions à des aménagements et infrastructures susceptibles de créer des nuisances ou de contrarier les projets de développement des riverains : Nimby, Lulu, Banana $^{10}$, etc. Souvent connotés négativement, ils sont censés illustrer l'expression de comportements égoïstes d'individus ou de groupes face à un intérêt collectif qui s'incarnerait dans les projets publics. Afin d'apporter un regard plus informé sur les motifs de la conflictualité et sur les raisons qui poussent les populations à s'opposer à des projets jugés importants par l'État ou par des collectivités locales, il est nécessaire d'investiguer davantage leurs caractéristiques et leur mode de réaction à des décisions jugées inacceptables. Nous nous attachons maintenant à questionner les conditions de la contestation des projets d'infrastructures d'utilité publique. Cela suppose de caractériser successivement ces conflits au regard des procédures institutionnelles et politicoadministratives de la décision publique, de la démocratisation des choix publics qui ne réduit pas la contestation, voire lui donne davantage de possibilités d'expression avant d'esquisser un modèle explicatif des conflits d'infrastructure.

10. Respectivement : Not In My Back Yard, Locally Unwanted Land-Use, Build Absolutely Nothing Anywhere Near Anything. 
Tableau 3. Les étapes de la procédure de mise en œuvre d'un projet d'infrastructure d'utilité publique

\begin{tabular}{c|c|c|c|c}
\hline $\begin{array}{c}\text { Débat public } \\
\text { (le cas échéant) }\end{array}$ & $\begin{array}{c}\text { Etude quantitative (étude } \\
\text { d'impact, calcul économique public) }\end{array}$ & $\begin{array}{c}\text { Enquête } \\
\text { publique }\end{array}$ & $\begin{array}{c}\text { Déclaration } \\
\text { d'utilité } \\
\text { publique }\end{array}$ & $\begin{array}{c}\text { Mise } \\
\text { en service } \\
\text { du projet }\end{array}$ \\
\hline \multicolumn{2}{c|}{ Consultation - Négociation - Décision } & \multicolumn{3}{c}{ Contestation - Conflit } \\
\hline
\end{tabular}

Source : les auteurs

\section{Procédure décisionnelle et contestations éventuelles : généralités}

La prise de décision d'aménagement d'une infrastructure par les Pouvoirs publics fait aujourd'hui en France l'objet d'un processus long et complexe.

Schématiquement, le processus comprend deux étapes, qui participent à l'appréciation de l'utilité publique : d'abord l'évaluation quantitative, réalisée en amont de la consultation du public, qui détermine par le calcul économique la rentabilité socioéconomique du projet et de ses variantes et fait apparaître un bilan des coûts et avantages; puis l'enquête publique et, lorsque l'importance du projet le justifie, le débat public. Dans cette deuxième séquence, il s'agit de réaliser un bilan qualitatif du projet à partir des observations recueillies auprès du public, puis de procéder à la déclaration d'utilité publique et de mettre en œuvre le processus de réalisation du projet, qui peut demander une modification des plans locaux d'urbanisme et des expropriations.

Le tableau ci-dessus (tableau 3), illustre les étapes de la procédure de mise en œuvre d'un projet d'infrastructure d'utilité publique et l'apparition éventuelle d'une contestation de sa pertinence, telle que la recherche empirique sur le contentieux les fait apparaître.

Il faut souligner que l'enquête publique permet aux citoyens d'accéder à une phase très importante, qui précède la déclaration d'utilité publique : ils disposent de la possibilité d'accéder aux dossiers techniques et d'exprimer leur avis auprès du commissaire enquêteur. Ouverte par arrêté préfectoral et portée à la connaissance du public par voie d'affichage en préfectures et en mairies, elle constitue également la source exclu- sive de recueil ex-ante des observations des riverains sur le projet.

Il importe de souligner que le commissaire enquêteur ne vérifie pas si la procédure de concertation a rempli la fonction d'outil d'aide à la décision publique, mais seulement si elle a été correctement mise en place et si l'on peut tirer une conclusion « raisonnable » des avis recueillis auprès du public. Sa mission n'est pas de faire un état des possibilités d'améliorer la décision, mais d'établir un bilan qualitatif des avis reçus au regard de l'intérêt et de l'impact du projet. Il ne peut donner qu'une appréciation relative : des risques de nuisance ou de dommage pour les habitants peuvent ainsi être traduits dans son avis comme des remarques peu contraignantes pour le décideur public. Par ailleurs, le Préfet est libre d'interpréter l'importance relative des avis positifs ou des remarques critiques ou réserves. De nombreux spécialistes estiment que l'enquête publique « demeure une procédure d'information et de consultation, sans pouvoir décisionnel conféré au public » (Hostiou et Hélin, 1993). En tout état de cause, même si le commissaire enquêteur rend un avis défavorable, ce dernier n'a pas pour effet de remettre en cause la réalisation du projet : il contraint cependant à déplacer la déclaration d'utilité publique, du Préfet ou du ministre vers le Conseil d'État ${ }^{11}$.

L'exemple de Maisse, comme beaucoup d'autres en France (voir par exemple Torre et al., op. cit.), montre que les conflits surgissent principalement après la déclaration d'utilité publique et peuvent perdurer après la réalisation du projet. Ils mettent généralement en question la fiabilité de l'étude

11. Sur ce point, voir Kirat et Levratto (2008). 
quantitative, ainsi que la régularité formelle du déroulement de l'enquête publique. Ils révèlent que les oppositions aux projets d'infrastructures découlent à la fois des caractéristiques de la procédure juridicoadministrative et de l'évaluation quantitative. Les divers acteurs concernés par un projet visent, en entrant dans un processus conflictuel, à faire annuler ou à infléchir un projet qui leur est défavorable en s'appuyant sur deux registres :

- le non-respect des étapes procédurales de la décision, en termes juridiques sa « légalité interne »: lacunes dans l'affichage des panneaux d'enquête publique, inaccessibilité du dossier d'étude d'impact ou son manque de clarté, non-disponibilité du commissaire enquêteur, ou encore absence des documents permettant une information complète du public. Ces éléments sont très fréquents dans l'argument des auteurs de recours devant le juge administratif ${ }^{12}$;

- les éléments non valorisables ignorés par le calcul économique, concernant notamment les principes d'équité ainsi que les impacts sur l'environnement ou sur le cadre de vie des résidents. Le développement du droit de l'environnement favorise cette stratégie, puisque les riverains se saisissent de ses éléments pour s'opposer à certains projets d'infrastructures.

Il est possible de dresser un profil idéaltypique d'un conflit d'infrastructure porté devant la juridiction administrative : le conflit prend naissance sur la base d'un acte administratif, la déclaration d'utilité publique, formulée par l'administration au nom de l'intérêt général. Des acteurs locaux s'opposent alors au projet et attaquent cette déclaration au nom des nuisances avérées, supposées ou anticipées du projet au niveau local. De telles manifestations ne sont pas rares et posent la question du bien-fondé des différends. D'où vient le problème ?

12. On peut espérer que la loi Grenelle II corrigera ces défauts grâce à la légalisation des sites de dépôt d'avis en ligne.
Pourquoi la déclaration d'utilité publique est-elle contestée, même lorsqu'elle a été précédée par de longues négociations entre élus, entreprises, riverains, et que le projet a fait l'objet d'une enquête publique, voire d'un débat public ? ${ }^{13}$

\section{Démocratisation des choix publics et persistance des conflits}

La « démocratisation » des projets publics (Blatrix, 1997, 2009a, 2009b ; Blatrix et al., 2007) a renouvelé, depuis les années 1980 , le contexte de la décision publique. L'ouverture du processus décisionnel vers les citoyens se traduit par un processus de plus et plus long, où le décideur permet aux acteurs de prendre la parole ${ }^{14}$. L'État n'a cessé, depuis quinze ans, de multiplier les procédures qui permettent au public de donner son avis. Une règle implicite semble présider à ces innovations successives : chaque procédure nouvelle va plus en amont que la précédente, descend plus avant dans les considérations techniques et prend plus de temps.

En effet, les premières enquêtes publiques avaient pour but de protéger les propriétaires contre les empiètements abusifs de l'Administration. Le projet technique était déjà là, finalisé, indiscutable ; la discussion se faisait à la marge. La loi $\mathrm{n}^{\circ} 83-630 \mathrm{du} 12$ juillet 1983 relative à la démocratisation des enquêtes publiques et de la protection de l'environnement (dite loi Bouchardeau), a fait évoluer la procédure, puisque le commissaire enquêteur enregistre désormais les avis, qui peuvent déborder le cadre étroit de la solution technique proposée.

13. Pour les grands projets, la création de la Commission nationale du débat public (CNDP) en 2002 a permis aux décideurs de mieux comprendre les intérêts des acteurs concernés et de débattre en amont de la réalisation du projet.

14. Dans un autre domaine, celui de la gestion de l'eau sur les bassins versants, Borowski et al. (2008) montrent que l'intégration de la participation du public aboutit à des incohérences institutionnelles et spatiales. 
C'est ce débordement qui pousse, en 1992, à la mise en place d'une nouvelle procédure, dite circulaire Bianco, propre aux autoroutes et aux voies ferroviaires. Avec cette formule, la discussion est ouverte bien avant la phase du tracé qui sera soumis ultérieurement à l'enquête publique.

La convention d'Aarhus portant sur l'accès à l'information, la participation du public au processus décisionnel et l'accès à la justice en matière d'environnement, entrée en vigueur en 2001, a modifié de façon importante l'encadrement de la décision publique en matière d'environnement. Cette convention, qui prolonge les déclarations de Stockholm de 1972 et du Sommet de Rio de 1992, crée le cadre légal pour la participation publique dans des projets territoriaux. Désormais, le droit interne français est subordonné à la convention internationale, qui prévoit la participation publique «tout au long du processus décisionnel »(Article 6).

Les impacts de cette adoption sont rapidement visibles. D'une part, elle pousse plus loin la décentralisation de l'État au regard de ses compétences en matière d'environnement et d'urbanisme. La loi du 27 février 2002 relative à la démocratie de proximité (codifiée par les articles L121-1 à L121-13 du code de l'environnement) institue alors une commission nationale du débat public en tant qu'entité administrative indépendante, chargée « de veiller au respect de la participation du public au processus d'élaboration des projets d'aménagement ou d'équipement d'intérêt national de l'État, des collectivités territoriales, des établissements publics et des personnes privées, relevant de catégories d'opérations dont la liste est fixée par décret en Conseil d'État, dès lors qu'ils présentent des forts enjeux socio-économiques ou ont des impacts significatifs sur l'environnement ou l'aménagement du territoire ».

D'autre part, elle constitue les fondements des lois Grenelle I et II, qui tout en statuant sur les questions d'environnement, renforcent des éléments procéduraux de l'enquête publique, notamment dans la prise en compte de l'avis du commissaire enquêteur par l'administration en charge du projet. Il est désormais légalement possible de demander l'annulation d'un projet ayant reçu l'avis défavorable du commissaire enquêteur.

De nombreux travaux en sciences sociales, les plus récents du groupe GIS Participation et Démocratie ${ }^{15}$, révèlent des « failles » dans le mécanisme décisionnel (Blondiaux et Fourniau, 2011), particulièrement le caractère inachevé d'une véritable participation publique dans le processus décisionnel : «la démocratisation du processus de décision n'est guère au rendez-vous » (Bherer, 2011), «la plupart des procédures sont sans effet juridique sur la décision » (Monédiaire, 2011), «les asymétries de pouvoir et de savoir entre les acteurs ne se réduisent pas sensiblement » (Blondiaux et Fourniau, 2011). Les conflits qui découlent de la décision publique et la discussion sur les problèmes posés par la procédure de concertation publique commencent alors à prendre de l'ampleur, en particulier dans le cadre des recherches menées sur la place du débat public et de la démocratie participative dans l'aménagement du territoire. Pourtant, l'opposition de la population à des projets d'infrastructure est encore souvent regardée comme la manifestation d'une logique individualiste exacerbée, qui irait à l'encontre de l'utilité publique. C'est du moins la vision de la décision publique « traditionnelle », où le décideur se dote d'un pouvoir absolu afin de réaliser le projet au nom de l'intérêt général.

Le principe selon lequel les intérêts particuliers doivent s'effacer devant l'intérêt général est central dans l'idéologie et la pratique du droit public français, comme dans la conception de la place de l'État dans la société. La traduction économique de cet intérêt général réside dans le concept de

15. http://www.participation-et-democratie.fr 
rentabilité socio-économique, issu du calcul économique public. Or, ni la rationalité administrative ni le calcul économique ne garantissent totalement l'équité dans les projets publics.

La décision publique repose, en France tout au moins, sur la tradition du calcul économique public, qui se fonde sur une évaluation des impacts attendus des projets proposés. Fruit des recherches des ingénieurs économistes français à l'École des Ponts-et-Chaussées (Guesnerie, 2006), il reflète la volonté du décideur public de réaliser un bilan économique des impacts de ses investissements présents et futurs. Utilisant des techniques mathématiques et d'ingénierie pour déterminer les caractéristiques de l'utilité publique, le calcul a longtemps été considéré comme la solution de référence pour justifier du caractère optimal de la décision de construction d'infrastructures publiques. En effet, ces dernières se caractérisent par une absence de marché, qui empêche de leur attribuer une valeur marchande et donc de déterminer les préférences des agents en fonction de leurs offres et demandes (Lebègue, 2005).

Le calcul économique public a vocation d'éclairer le choix politique en termes de bilan socio-économique ; il ne se limite pas à une simple étude de la rentabilité financière de l'investissement public. Mais ce point fait depuis longtemps débat, en particulier face aux problématiques d'économie du bienêtre et aux changements dans les demandes adressées aux décideurs. D'où des doutes relatifs aux méthodes et techniques de calcul, pas toujours appropriées pour piloter les choix de société contemporains. Les critiques les plus importantes portent toutefois sur l'identification et la valorisation des effets du projet et de leur validation ou de leur acceptation par le public, en termes de mesure des impacts non monétarisables ou de compensation des nuisances (Pham et Torre, 2012).

Il en résulte deux difficultés majeures. La première concerne l'arbitrage des impacts et l'allocation des droits et nuisances résultant des projets proposés, qu'il s'agisse de l'arbitrage entre secteurs différents ou entre périodes présente et futures. Par exemple, dans le cas de construction d'une route, l'absence de marché interdit toute évaluation incontestable d'impacts qualitatifs comme la modification d'un paysage remarquable ou la diminution des accidents de la circulation, et encore plus leur estimation future. De même, toutes les nuisances ne sont pas quantifiables : comment monétariser exactement le désagrément dû à la proximité d'éoliennes ou de lignes à haute tension ? D'où la difficulté de compensations justes, qui se heurte également au fait que certains acteurs font apparaître après la mise en œuvre du projet (de nouveaux résidents d'une commune par exemple). La seconde difficulté tient au traitement équitable des acteurs concernés par le projet. Souvent issus de catégories socioprofessionnelles différentes, ils reflètent des conceptions et des opinions divergentes (Crozet, 2004, 2006).

L'étude des conflits montre que depuis l'ouverture de la décision publique, la difficulté d'arbitrage, particulièrement entre des intérêts non monétarisables, est devenu l'enjeu principal du décideur public ${ }^{16}$. La conversion en valeur monétaire des impacts d'un projet se heurte à des obstacles sérieux, en particulier les aspirations de la population en termes de valeur sociale, exprimées comme des revendications de droits subjectifs : jouir d'un cadre de vie paisible, protéger l'environnement, éviter les nuisances industrielles ou sonores ou encore participer à la prise de décision.

Les conflits d'infrastructures montrent que le processus décisionnel public conduit souvent à affirmer le caractère d'utilité publique d'un projet sans pour autant apporter

16. On ne sait pas, par exemple, quelle est la valeur à primer dans l'exemple de Maisse : l'environnement ou la sécurité routière ? La réponse dépend beaucoup de la prise de position de chacun dans l'histoire. 
la preuve qu'il s'agit de la solution la plus satisfaisante pour la population, d'où le sentiment des opposants d'être souvent victimes d'une « mauvaise » décision (Raux et al., op. cit.). Tout se passe comme si les limites du calcul économique étaient compensables par la consultation du public, et vice versa. L'Administration s'appuie sur le calcul économique pour ouvrir l'enquête publique, pour ensuite valider ce calcul par les résultats de l'enquête : il s'agit donc d'un schéma circulaire dans lequel on peut très bien arriver à valider un calcul « erroné » par une consultation non exhaustive de la population, d'autant plus que les variantes du projet ne sont pas ouvertes à la discussion publique. L'enquête publique impose des contraintes sur le maître d'ouvrage mais elle ne permet pas de remettre en cause l'opportunité de la décision d'aménagement.

Ajoutons à cela que seule l'expropriation pour cause d'utilité publique fait obligatoirement l'objet d'une compensation ; la dévalorisation des propriétés exposées aux nuisances ne fait pas partie des préjudices indemnisables. Enfin, la place du juge administratif reste circonscrite à la légalité « interne » et « externe ${ }^{17}$ de la décision contestée, même si la «théorie du bilan » peut laisser penser qu'il pèse les intérêts en présence et procède à une évaluation propre des coûts et avantages du projet sur lequel porte une déclaration d'utilité publique. En effet, selon la jurisprudence administrative (Conseil d'État, Ville Nouvelle Est, 28 mai 1971), dite « théorie du bilan », une opération ne peut légalement être déclarée d'utilité publique que si les atteintes à la propriété privée, le coût financier et éventuellement les inconvénients d'ordre social et les atteintes à d'autres intérêts publics qu'elle comporte ne sont pas excessifs eu égard à l'intérêt

17. La légalité interne concerne la question de savoir s'il y a eu violation de la loi, sous la forme d'erreurs de droit, de qualification juridique ou de fait; la légalité externe répond à la question de savoir si la décision prise l'a été de manière régulière, indépendamment de son contenu. qu'elle présente. Cet arrêt fut innovant dans la mesure où il élargit la sphère d'appréciation de l'utilité publique : désormais, le juge s'attache à déterminer si le coût financier de l'opération et les inconvénients qu'elle implique ne sont pas disproportionnés relativement à ses avantages. Cependant, l'application de la théorie du bilan n'est pas sans limites, d'abord parce que le juge n'a pas compétence pour statuer sur l'opportunité du choix par l'administration d'une localisation ou d'un tracé parmi un ensemble d'alternatives, ensuite parce que la « grille d'analyse » de l'utilité publique fondée sur une mise en balance des avantages et des coûts et inconvénients est peu élaborée et, selon le Conseil d'État, a débouché sur un nombre relativement limité d'annulations ${ }^{18}$.

\section{Un cadre de compréhension des conflits liés à la décision publique}

Dans le domaine des infrastructures, comme d'ailleurs dans celui des activités industrielles ou extractives réglementées, les conflits portés au contentieux concernent essentiellement le projet et non le bien ou l'équipement déjà mis en fonctionnement, et visent à empêcher sa réalisation. Par ailleurs, que soient mobilisés le registre de l'environnement ou la contestation des procédures de décision, c'est bien du cadre de vie dont il est question : les riverains cherchent à défendre leur environnement, qui leur semble menacé par la procédure qui se met en place, en s'appuyant sur les ressources offertes par le droit, quand il ne s'agit pas de manifestations ou de mobilisation des médias.

Comme suggéré par la littérature (Abrantes et al., 2010 ; Pham et Kirat, op. cit. ; Torre et al., op. cit.) la dynamique d'occupation des sols et de transformation des usages constitue une cause importante et quasi mécanique des conflits d'usage de l'espace, et tout particulièrement des conflits

18. http://www.conseil-etat.fr/cde/node.php? articleid $=1307$ 
d'infrastructures, ne serait-ce que par l'avancée irrésistible des fronts d'urbanisation dans des régions comme l'Île-deFrance. Mais ce n'est pas la seule variable explicative de la conflictualité, qui trouve aussi ses racines dans les comportements différenciés des populations locales face aux projets, ainsi que dans les caractéristiques morpho-spatiales des territoires concernés. En effet, l'apparition et les modalités de déroulement des conflits d'usage sont également déterminés par les paramètres socioéconomiques caractéristiques de l'espace des projets de transformation des usages des sols, qu'il s'agisse d'espaces localisés ou d'espaces linéaires (O'Lear et al., 2005, ou Wester-Herber, 2004).

Certains géographes urbains ont adopté ce point de vue (Joerin et al., 2005 ; Trudelle, op. cit.). Ils reprennent l'approche sociologique du conflit - relation antagonique entre deux ou plusieurs unités d'action dont l'une au moins tend à dominer le champ social de leurs rapports - cause et fruit d'un changement relationnel et fortement lié à la perturbation des valeurs sociales. Mais ils reconnaissent également ce que nous qualifions de dimension «matérielle » du conflit et qui suppose son intégration dans un cadre spatialisé. Tensions et différends n'apparaissent pas par hasard sur le territoire: ils sont dépendants du contexte matériel des territoires occupés, qu'il s'agisse de caractéristiques géophysiques (plaine, montagne, littoral), spatiales (rural, urbain, périurbain) ou sociodémographiques (structure par âge ou par CSP de la population, solde démographique). Le travail empirique se doit de chercher à comprendre et à généraliser les caractéristiques de la conflictualité mais aussi le rapport qu'elle entretient avec son espace.

L'étude du conflit peut être aussi menée dans le cadre plus large de l'analyse du processus de décision publique. Dans un contexte où de plus en plus de décisions publiques en matière d'aménagement de territoire sont contestées en France, elle révèle à quel point ces procédures sont longues et peu efficaces. Certains aménageurs connaissent cette faiblesse depuis longtemps mais considèrent que le problème vient de la façon dont le projet est publiquement présenté. "La question qui se révèle est finalement moins de l'ordre méthodologique que de la capacité à convaincre de l'utilité du projet » (Mériaux et al., 2002). Pourtant, la complexification de la procédure décisionnelle ne conduit pas à davantage d'acceptation locale, et l'on peut constater que les contestations visant directement la procédure sont de plus en plus nombreuses. En effet, si les riverains sont affectés par la décision publique, chercher à les convaincre sera chose impossible. La question sera plutôt de révéler les préjudices, et d'ajuster la décision devant ces nouvelles variables. L'étude des conflits ouvre alors une piste de réflexion sur ces possibilités. Les travaux de Torre et al. (op. cit.) et Bouba-Olga et al. (op. cit.) sur la capacité des territoires pour prendre la parole devant une décision d'aménagement, et de Jeanneaux (op. cit.) sur le pouvoir d'ajustement de la décision publique des conflits d'usage, vont dans ce sens.

Pour tenter de comprendre la place prise par les conflits dans le processus de développement territorial ainsi que le rôle qu'ils occupent dans l'expression des populations, nous proposons d'utiliser l'analyse d'Hirschman (1970), qui pose la question des réactions à un choc non désiré selon le triptyque exit, voice, loyalty. Cette approche offre en effet un cadre de compréhension des modes de conflictualité, en particulier dans le cas des conflits d'infrastructures.

Quand se produit un événement qui laisse insatisfait un agent (ou un groupe d'acteurs) ${ }^{19}$,

19. Le terme d' «événement» ne recouvre pas exclusivement des actes techniques avérés, tels que la construction d'un immeuble ou d'une bretelle d'autoroute, un remembrement, la pollution d'une rivière... ; il peut également s'agir d'une décision censée avoir des effets dans le futur. 
trois types de solutions se présentent, qui constituent autant de réponses à la situation :

- les acteurs qui considèrent que l'événement est non conforme à leurs intérêts peuvent décider de la solution d'exit. Il s'agit d'une stratégie de sortie, qui consiste, soit à quitter le territoire et à se localiser dans une zone jugée plus favorable (l'exit spatial est alors un « vote avec les pieds »), soit à quitter son activité économique et à se reconvertir dans une activité compatible avec les nouvelles conditions. Il va sans dire que cette solution n'est pas toujours praticable, pour des raisons financières ou de prix du foncier (par exemple, il est difficile de revendre à un bon prix des terrains menacés par l'arrivée d'une infrastructure de transport, d'autant plus qu'en France le préjudice économique n'est pas compensable en dehors d'une expropriation pour cause d'utilité publique). De même, seuls les acteurs expropriés sont susceptibles de recevoir une indemnisation, en aucun cas automatique pour les riverains concernés ;

- une autre solution consiste à adopter une attitude loyale à l'égard du processus en cours, c'est la loyalty. Nous l'interprétons comme l'acceptation des décisions en cours, par une participation aux dynamiques et aux projets mis en place, ainsi que par l'absence d'opposition publique. Une des manières de procéder est alors d'attendre les élections suivantes, afin de présenter un programme concurrent et tenter de se faire élire. Cette solution implique une fidélité à l'action publique, peu compatible avec les recours contre le processus de décision publique ;

- la troisième solution est celle du voice. Il s'agit de faire entendre sa voix et de s'opposer ainsi aux projets, aux décisions ou aux actions en cours. C'est alors la voie du conflit qui est adoptée. Il convient de distinguer entre le voice individuel, plutôt réservé aux petits conflits, et le voice collectif, qui marque un élargissement de l'espace de «concernement », ainsi que la mobilisation contre des projets importants, ou qui impliquent une reconfiguration forte des modalités de gouvernance au niveau local (Dowding et al., 2000). Dans ce dernier cas, le processus de voice est souvent dirigé vers les pouvoirs publics (Young, 1976), avec l'idée de remettre en question les décisions prises par les autorités et d'infléchir le processus de gouvernance.

$$
\text { * * }
$$

L'objectif de cet article était de présenter et d'analyser les conflits d'infrastructures en Île-de-France et, au-delà, de jeter un éclairage sur le processus de décision publique en la matière, ainsi que sur le rôle joué par les conflits dans ce processus. Nous avons en particulier conduit cette étude afin de mieux comprendre ce que l'on identifie parfois comme des «problèmes ». À savoir la persistance, voire l'accentuation des conflits, et plus précisément des recours contentieux, en dépit des évolutions des enquêtes publiques vers une consultation élargie du public et une association plus importante des populations à la décision en matière de projets d'infrastructures.

L'étude appliquée, menée sur les données du contentieux, nous a permis de mettre en évidence un certain nombre d'élément saillants. Les conflits d'infrastructures en Région Île-de-France sont essentiellement localisés dans les espaces périurbains, dans des communes à la population relativement aisée, et font écho à la lutte vive entre besoins d'urbanisation et de préservation de l'espace. Ils expriment des contestations locales de riverains soucieux de la protection et des atteintes à leur cadre de vie, qui s'opposent à des projets considérés comme utiles à l'intérêt général par leurs porteurs (généralement les Pouvoirs publics), mais qui ne leur paraissent pas acceptables en l'état. Ils révèlent enfin que tous les territoires n'ont pas la même capacité de réaction à l'installation des infrastructures, en raison des 
différences socioéconomiques des populations qui les occupent et de leur capacité à faire entendre leur voix.

Nous avons également pu éclairer le principe de la décision publique et montrer que la présence des conflits correspond à des imperfections dans le mécanisme de prise de décision et à une insatisfaction persistante des populations locales quant à l'expression de leurs revendications. Au total, il nous semble que l'analyse des conflits peut reposer sur une réinterprétation de l'approche Hirshmanienne, dans laquelle le conflit correspond au voice et révèle la capacité des habitants à exprimer à la fois leur opposition aux projets proposés et leur implication dans le débat local, une position bien éloignée des attitudes de passivité ou d'abandon du territoire exprimées par l'exit ou la loyauté.

Si la concertation préalable améliore l'information du décideur public, il serait abusif, voire erroné, de soutenir qu'il est possible d'arbitrer les intérêts particuliers par le seul truchement des négociations, en raison de l'existence possible d'intérêts incompatibles. C'est ici que les conflits entrent en jeu. En effet, il ne suffit pas, pour un particulier ou une association, d'être présent lors de l'enquête publique pour négocier de manière efficace. Il importe également de prendre position dans le débat, de faire entendre sa voix, et de s'accorder avec d'autres groupes sur des valeurs défendables en commun, grâce à quoi il devient possible de jouer un rôle dans la « fabrication » d'un projet et d'infléchir le processus de décision publique.

Enfin les conflits d'infrastructures mettent en évidence la complexité mais aussi les ambiguïtés du processus de décision publique, qui se trouve confronté à un double dilemme : l'arbitrage entre les dimensions qualitatives et quantitatives et la difficile soudure entre le travail réalisé au sein de l'administration et les procédures d'enquêtes publiques. Vient s'ajouter, à cet équilibre complexe, la question des échelles spatiales. Quelle est l'échelle pertinente, en matière de décision publique, entre les exigences d'espace d'une Région Capitale et les attentes de territoires de tailles plus restreintes mais dans lesquels vivent des populations fermement décidées à défendre leur environnement et leur cadre de vie ?

Les auteurs désirent remercier deux rapporteurs anonymes pour leurs commentaires très riches, qui ont conduit à une refonte complète de l'article. Toutefois, ils restent seuls responsables des erreurs ou omissions pouvant subsister dans le présent texte. 


\section{RÉFÉRENCES BIBLIOGRAPHIQUES}

Abrantes P., Soulard C., Jarrige F., Laurens L. (2010). Dynamiques urbaines et mutations des espaces agricoles en LanguedocRoussillon. Cybergeo: European Journal of Geography [En ligne], Espace, Société, Territoire, document 485, mis en ligne le 13 janvier 2010, consulté le 02 novembre 2011.

URL : http://cybergeo.revues.org/22869; DOI : $10.4000 /$ cybergeo. 22869

Bherer L. (2001). Les relations ambiguës entre participation et politiques publiques. Participations, $\mathrm{n}^{\circ}$ 1, p. 105-133.

Blatrix C. (1997). Faut-il un public à l'enquête publique ? Les paradoxes d'une procédure de démocratie participative. Techniques, Territoires et Sociétés, $\mathrm{n}^{\circ} 34$, p. 101-110.

Blatrix C. (2009a). Cadrages, recadrages et hors-champ du débat public : le cas de l'assainissement en Île-de-France. In «Ville éphémère, ville durable. Nouveaux usages, nouveaux pouvoirs », Paris, L'œil d'or.

Blatrix C. (2009b). La démocratie participative en représentation. Sociétés contemporaines, $\mathrm{n}^{\circ}$ 74, p. 97-119.

Blatrix C., Blondiaux L., Fourniau J.-M., Heriad-Dubreil B., Lefebvre R., Revel M. (2007). Le débat public : une expérience française de démocratie participative. Paris, La Découverte.

Blondiaux L., Fourniau J.-M. (2011). Un bilan des recherches sur la participation $\mathrm{du}$ public en démocratie : beaucoup de bruit pour rien ? Participations, vol. $1, \mathrm{n}^{\circ} 1$, p. 8-35.

Borowski I., Le Bourhis J.-P., Pahl-Wostl C., Barraqué B. (2008). Spatial misfit in participatory river basin management: effects on social learning. A comparative analysis of German and French case studies. Ecology and Society, vol. 13, $\mathrm{n}^{\circ} 1$, http://www.ecologyandsociety.org/vol13/ iss $1 / \operatorname{art} 7 /$
Bouba-Olga O., Chauchefoin P. (2001). Le conflit de la surface et du réseau : proximité géographique et proximité économique. Annales de la Recherche Urbaine, $\mathrm{n}^{\circ}$ 90, p. 7-14.

Bouba-Olga O., Boutry O., Rivaud A. (2009). Un approfondissement du modèle ExitVoice par l'Économie de la Proximité. Nature, Sciences et Sociétés, $\mathrm{n}^{\circ} 17$, p. 381-390.

Bossuet L. (2007). Les conflits du quotidien en milieu rural. Géographie, Économie et Société, $\mathrm{n}^{\circ} 3$, p. 141-164.

Cadoret A. (2006). Conflits d'usage lié à l'environnement et réseaux sociaux: enjeu d'une gestion intégrée - Le cas du littoral $d u$ Languedoc-Roussillon. Thèse de Doctorat en géographie, Université de Montpellier III, $586 \mathrm{p}$.

Caron A., Torre A. (2006). Quand la proximité devient source de tensions : conflits d'usages et de voisinage dans l'espace rural. Développement Durable et Territoires, $\mathrm{n}^{\circ} 7$, revue en ligne.

Charlier B. (1999. La défense de l'environnement : entre espace et territoire. Géographie des conflits environnementaux déclenchés en France depuis 1974. Thèse pour le doctorat en géographie, Université de Pau et des pays de l'Adour.

Crozet Y. (2004). Calcul économique et démocratie : des certitudes technocratiques au tâtonnement politique. Cahier d'Economie Politique, vol. 2 ; $\mathrm{n}^{\circ} 47$, p. 155-172.

Crozet Y. (2006). Calcul économique et décision publique. Infrastructures \& Mobilité, vol. 59, p. 1-7.

Darly S. (2009). Faire coexister ville et agriculture au sein des territoires périurbains. Antagonismes localisés et dynamiques régionales de la conflictualité. Analyse du cas de l'île-de-France. Thèse de Doctorat en géographie, EHESS, Paris, $480 \mathrm{p}$. 
Darly S., Torre A. (2008). Conflits liés aux espaces agricoles et périmètres de gouvernance en Île-de-France (résultats à partir d'analyses de la presse quotidienne régionale et d'enquêtes de terrain). Geocarrefour, vol. 83, n 4, p. 307-319.

Dowding K., John P., Mergoupis T., Vught M.V. (2000). Exit, Voice, Loyalty: analytic and empirical developments. European Journal of Political Research, vol. 37, p. 469-495.

Gilly F. (2007). Paris, une métropole dans le monde, mise en perspective des savoirs. Etude exploratoire pour la DIACT, Université de Lille, 102 p.

Guesnerie R. (2006). De l'utilité du calcul économique public. Économie \& Prévision, ${ }^{\circ}$ 175-176, p. 1-15.

Hirschman A.-O. (1970). Exit, Voice and Loyalty - Responses to decline in firms, organizations and states. Cambridge (MA), Harvard UniversityPress, 176 p.

Hostiou J.-C., Hélin R. (1993). Droit des enquêtes publiques. Ed. Le Moniteur, $393 \mathrm{p}$.

IAURIF (2001). Mode d'occupation du sol. Note rapide, $\mathrm{n}^{\circ} 282$, juillet.

Jeanneaux P. (2006). Les conflits d'usage dans les zones périurbaines et rurales françaises - Une approche par l'analyse économique de la décision publique. Thèse de doctorat en Sciences Économiques, Université de Bourgogne, Dijon, 240 p.

Jeanneaux P., Perrier?Cornet P. (2009). La contestation locale des décisions publiques et les conflits d'usage dans les espaces ruraux et périurbains français. Économie Appliquée, vol. LXII, n4, p. 157?182.

Joerin F., Pelletier M., Trudelle C., Villeneuve P. (2005). Analyse spatiale des conflits urbains. Enjeux et contextes dans la région du Québec. Cahiers de Géographie du Québec, vol. 49, n ${ }^{\circ}$ 138, p. 319-342.

Kirat T., Levratto N. (2008). Tous égaux face aux nuisances des infrastructures de transport ? Le calcul économique et le droit administratif au défi de l'équité.
Géographie, Économie, Société, vol. 10, $\mathrm{n}^{\circ} 3$, p. 350-364.

Kirat T., Melot R. (2006). Du réalisme dans l'analyse économique des conflits d'usage : les enseignements de l'étude du contentieux dans trois départements français (Isère, Loire-Atlantique, SeineMaritime). Développement Durable et Territoires, Dossier 7: Proximité et environnement,

http://developpementdurable.revues.org/do cument2574.html

Kirat T., Torre A. (dir.) (2006). Conflits d'usage et dynamiques spatiales : les antagonismes dans l'occupation des espaces ruraux et périurbains » (I), Géographie, Économie, Société, vol. 8.

Lascoumes P., Le Bourhis J.-P. (1998). Le bien commun comme construit territorial, identités d'action et procédures, Politix, $\mathrm{n}^{\circ} 42$, p. 37-66.

Lebègue D. (2005). Révision du taux d'actualisation des investissements publics. Rapport du Commissariat Général du Plan, $112 \mathrm{p}$.

Lecourt A. (2003). Les conflits d'aménagement - Analyse théorique et pratique à partir du cas de Breton. Thèse de Doctorat en Géographie, Université de Rennes II, Rennes.

Mathieu N.-H (2005). La population francilienne au $1^{\mathrm{er}}$ janvier 2005. Document de l'INSEE - Ille-de-France - Faits et Chiffres, $\mathrm{n}^{\circ} 112,2006,4 \mathrm{p}$.

Mélé P., Larrue C., Rosemberg M. (2003). Conflits et Territoires. Tours, PUF, $224 \mathrm{p}$.

Melot R. (2009). De la gestion des espaces au projet de territoire : les enjeux politiques d'un changement de paradigme juridique. L'Année sociologique, vol. 59, $\mathrm{n}^{\circ} 1$, p. 177-199.

Meriaux A., Kast R., Lapied A. (2002). Prévoir l'impact d'un projet d'équipement. Economie publique, vol. 1, n 10, p. 39-49.

Monédiaire G. (2011). La participation du public organise par le droit : des principes prometteurs, une mise en œuvre circonspecte. Participation, $\mathrm{n}^{\circ}$ 1, p. 134-155. 
O’Lear S., Diehl P. F., Frazier D.-V., Allee T.-L. (2005). Dimensions of territorial conflict and resolution: tangible and intangible values of territory, GeoJournal, $\mathrm{n}^{\circ}$ 64, p. 259-261.

Orizet F.-R. (2003). Mobilité - Réflexions sur l'avenir de l'Île-de-France. Direction Régionale de l'Equipement, 92 p.

Pham H.-V., Kirat T. (2008). Les conflits d'usage des espaces périurbains et le contentieux administratif - Le cas de la Région Île-de-France. Revue d'Économie Régionale et Urbaine, $\mathrm{n}^{\circ}$ 5, p. 671-700.

Pham H. V, Torre A. (2012). La décision publique à l'épreuve des conflits, un cadre d'analyse des processus décisionnels au regard de l'expression des oppositions. Revue d'Économie Industrielle, 138, p. 93-126.

Raux C., Souche S., Vaskova L. (2007). Les figures de l'acceptabilité. In « Le calcul économique public », Economica, p. 432-454. SEGESA/DREIF/DRIAF (2005). Dynamique territoriale de l'agriculture et de l'espace rural en Île-de-France. http://driaf.Ilede-France.agriculture.gouv.fr/IMG/pdf_ Partie1_Dynamiq_territor_agr_iIdF_ cle884a36.pdf.
Slak M.-F., Lee A., Michel P. (2001). L'évolution des structures d'occupation du sol vue par Teruti. Agreste Cahiers, $\mathrm{n}^{\circ}$ 1, mars, p. 13-25.

Torre A., Aznar O., Bonin M., Caron A., Chia E., Galman M., Lefranc C., Melot R., Guérin M., Jeanneaux P., Kirat T., Paoli J.C., Salazar M.-I., Thinon P. (2006). Conflits et tensions autour des usages de l'espace dans les territoires ruraux et périurbains. Le cas de six zones géographiques françaises. Revue d'Economie Régionale et Urbaine, vol. 3, p. 411-450.

Trudelle C. (2003). Au-delà des mouvements sociaux : une typologie relationnelle des conflits urbains. Cahiers de Géographie du Québec, vol. 47, n 11, p. 223-242.

Wester-Herber M. (2004). Underlying concerns in land-use conflicts-the role of place-identity in risk perception. Environmental Science \& Policy, $\mathrm{n}^{\circ} 7$, p. 109-116.

Young D.-R. (1976). Consolidation or diversity: choices in the structure of urban governance. The American Economic Review, vol. 66, $\mathrm{n}^{\circ} 2$, p. 378-385. 